\title{
Histologically surprising nasal polyps
}

Marina Boscolo, France Devuyst, Bernard Corvilain. CHU Erasme, ULB, Belgium

\section{INTRODUCTION}

Pituitary macroadenomas usually present with symptoms due to a local mass effect or to hormone abnormalities. Acromegaly is due an excessive growth hormone production, usually caused by a slow-growing pituitary adenoma.

Acromegaly is an insidious disease. An average delay of seven years is reported between the time of symptoms onset and diagnosis. According to main symptoms, acromegaly is usually diagnosed by internists, ophthalmologists or rheumatologists. It may be suspected by pneumologists in case of sleep apnea syndrome. Only exceptionally otorhinolaryngologists diagnose the disease, even if nasal polyps may be present in up to $5 \%$ of patients.

\section{CASE REPORT}

A 34-year-old woman presented to an otorhinolaryngologist with persistent nasal congestion and forehead pain, unresponsive to antibiotic and antiinflammatory treatment.

Nasal endoscopy confirmed the presence of nasal polyps. Computed tomography of paranasal sinuses showed massive mucosal hypertrophy with a voluminous polypoid intranasal lesion (Fig.1).

Transnasal biopsy demonstrated the presence of pituitary tissue, and gadolinium-enhanced magnetic resonance images confirmed the presence of a large pituitary mass (Fig.2).

Hormonal evaluation showed a very high serum IGF-I (1185 ng/ml; N: 104-380) and an increased serum growth hormone (48 ng/ml; $\mathrm{N}<3.5)$.

The patient underwent transphenoidal surgery and histology confirmed the presence of a GH secreting adenoma.

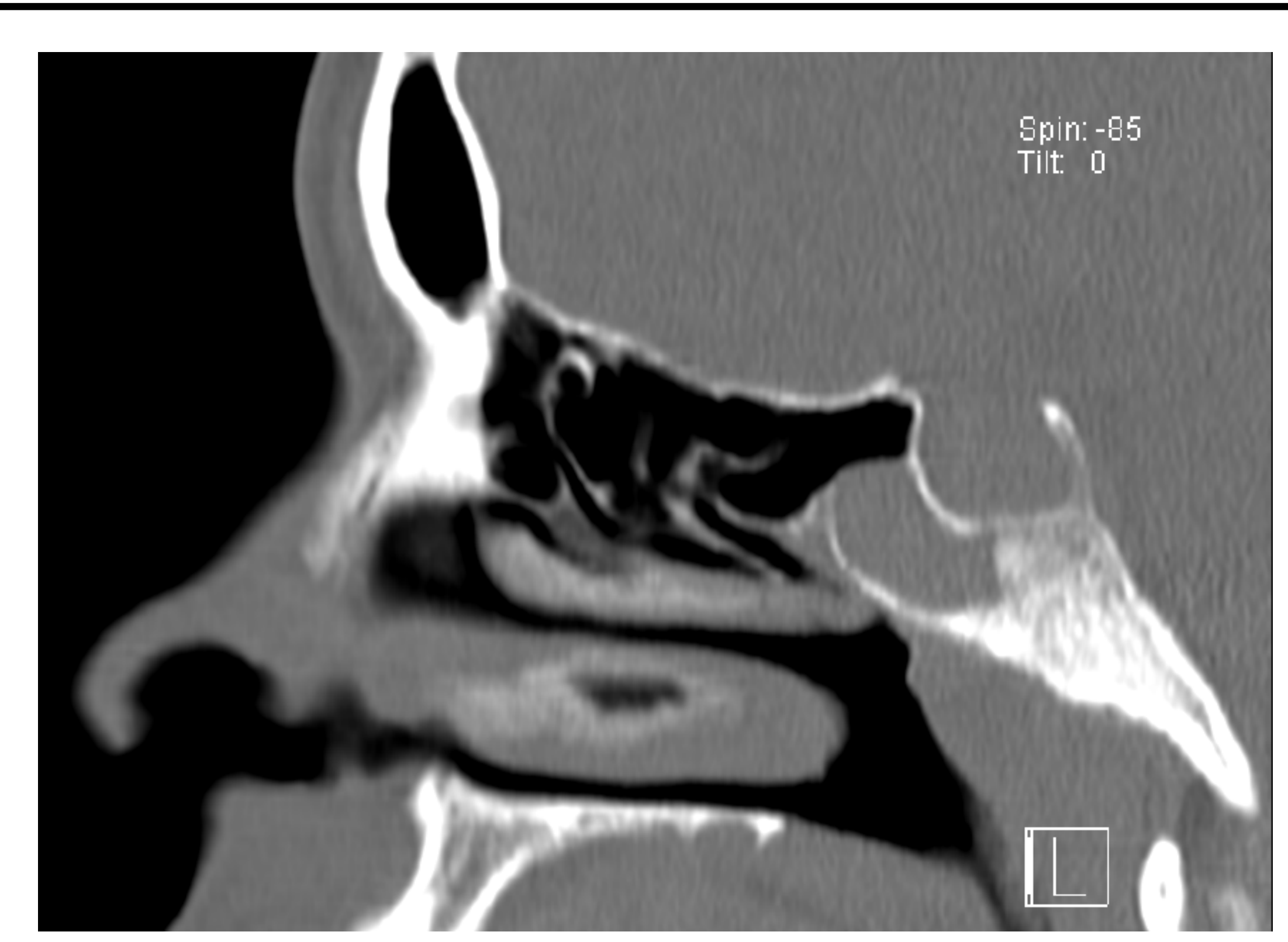

Fig.1 Computed tomography of paranasal sinuses showing massive mucosal hypertrophy with a voluminous polypoid intranasal lesion
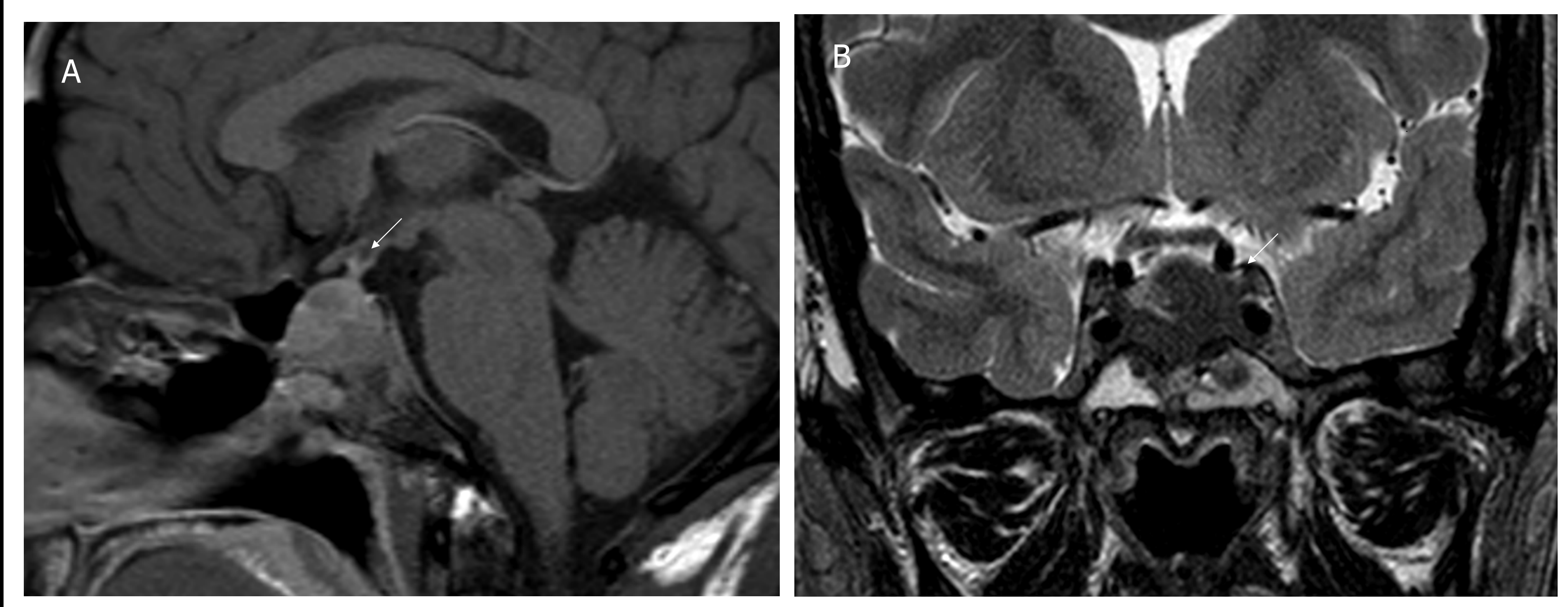

Fig.2 Gadolinium-enhanced MRI images focused on pituitary gland (a, coronal view; b, sagittal view)

\section{DISCUSSION}

This case illustrates that pituitary lesions may present as polyps invading nasal cavities. To our knowledge only ten cases have been reported in literature: seven macroprolactinomas, two non secreting adenomas and one macroadenoma with ACTH immunopositivity.

In all the reported cases initial diagnosis was that of a nasal polyp or of an intranasal carcinoma.

\section{CONCLUSIONS}

Intranasal presentations of pituitary tumours are uncommon. In a patient with nasal polyps, looking for clinical signs of Cushing syndrome or of acromegaly and subsequent hormonal evaluation could be of help for an early diagnosis.

\section{REFERENCES}

1. Johnston PC et al. Ir J Med Sci 2012; 181:277-279

2. Gnanalingham KK et al. British Journal of Neurosurgery 2003; 17:84

3. Lugo $G$ et al. 2011; 2012:1-10

4. Anagnostis $P$ et al. Int J Clin Pract 2011; 65:896-902

5. Drange MR et al. JCEM 1999; 85:168-174 